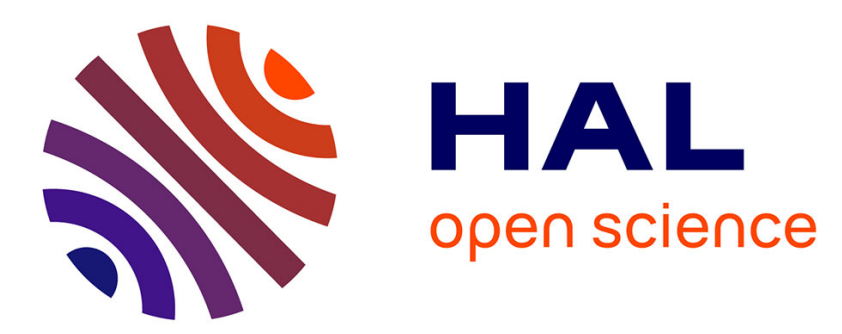

\title{
Influence de l'environnement et de la microstructure sur la propagation en fatigue des fissures courtes tridimensionnelles
}

J. Petit, A. Zeghloul

\section{- To cite this version:}

J. Petit, A. Zeghloul. Influence de l'environnement et de la microstructure sur la propagation en fatigue des fissures courtes tridimensionnelles. Revue de Physique Appliquée, 1989, 24 (9), pp.905913. 10.1051/rphysap:01989002409090500 . jpa-00246113

\section{HAL Id: jpa-00246113 https://hal.science/jpa-00246113}

Submitted on 1 Jan 1989

HAL is a multi-disciplinary open access archive for the deposit and dissemination of scientific research documents, whether they are published or not. The documents may come from teaching and research institutions in France or abroad, or from public or private research centers.
L'archive ouverte pluridisciplinaire HAL, est destinée au dépôt et à la diffusion de documents scientifiques de niveau recherche, publiés ou non, émanant des établissements d'enseignement et de recherche français ou étrangers, des laboratoires publics ou privés. 
Classification

Physics Abstracts

$46.30 \mathrm{~N}-62.20 \mathrm{M}$

\title{
Influence de l'environnement et de la microstructure sur la propagation en fatigue des fissures courtes tridimensionnelles
}

\author{
J. Petit et A. Zeghloul \\ Laboratoire de Mécanique et Physique des Matériaux, URA CNRS 863, ENSMA, 86034 Poitiers Cedex, \\ France
}

(Reçu le 2 février 1989, révisé le 7 juin 1989, accepté le 9 juin 1989)

\begin{abstract}
Résumé. - La propagation en fatigue de fissures courtes de surface a été étudiée dans un alliage léger à haute résistance type 7075 dans deux états de revenu, T651 et T7351. Les essais ont été effectués à l'air ambiant et sous vide, avec un rapport de charge $R=0,1$ et une fréquence de $35 \mathrm{~Hz}$. La longueur des fissures a été mesurée en surface à l'aide de répliques. Le comportement de ces fissures courtes tridimensionnelles (3D) a été comparé à celui de fissures bidimensionnelles (2D) traversantes courtes et longues étudiées précédemment. Les résultats sont discutés sur la base des mesures de fermeture effectuées sur les fissures longues et d'observations microfractographiques. La forte influence de l'environnement ambiant observée sur les fissures 3D s'avère comparable à celle observée précédemment pour les fissures $2 \mathrm{D}$ dans le domaine de vitesses exploré $<10^{-8} \mathrm{~m} /$ cycle. La moindre résistance à l'air est attribuée à un effet fragilisant de la vapeur d'eau. L'influence de la microstructure à l'échelle du grain au niveau du site d'amorçage s'avère très grande dans le cas d'une propagation cristallographique en stade I (7075 T651 sous vide); elle est par contre très réduite pour une propagation en stade II (7075 T651 à l'air, 7075 T7351 à l'air et sous vide). Une modélisation des effets de la plasticité localisée et de la microstructure est proposée.
\end{abstract}

\begin{abstract}
The propagation of small surface cracks has been studied on a high strength Aluminium alloy 7075 in peak aged (T651) and overaged (T7351) conditions. Tests were performed at a load ratio $R=0.1$ and a frequency of $35 \mathrm{~Hz}$ in ambient air and in vacuum. Small crack growth was monitored by mean of replicas. The behaviour of small cracks is compared to that of long and short through cracks and discussed on the basis of closure measurements performed on long cracks. A strong influence of environment on small crack growth has been shown which is similar to that observed for through cracks in the low rate range $\left(<10^{-8} \mathrm{~m} /\right.$ cycle $)$. The lower resistance observed in air has been related to water vapour embrittlement as previously shown for long cracks. The influence of microstructure at the scale of grain size has been shown to be large on crystallographic stage I propagation observed in vacuum on the $7075 \mathrm{~T} 651$, but weak on stage II propagation observed on the 7075 T651 in air and on the 7075 T7351 in both environmental conditions. A modelisation of localised plasticity and microstructure is proposed.
\end{abstract}

\section{Introduction.}

Les premiers travaux effectués sur l'influence de l'environnement sur la résistance en fatigue (durée de vie, vitesse de fissuration) avaient montré que, globalement, la résistance des métaux et alliages était plus faible à l'air ambiant que sous vide $[1,2]$. Plus récemment, des études conduites dans le domaine des basses vitesses de fissuration et près du seuil de propagation, sur des alliages légers à haute résistance ont montré comparativement au vide l'existence d'une forte influence de l'environnement ambiant sur la propagation des fissures longues [320].
Par ailleurs les fissures courtes ont constitué un thème privilégié de recherche au cours de la dernière décennie [21,22] suite aux travaux précurseurs de Pearson [23], qui en 1975, avaient montré un comportement «anormal» des fissures courtes par rapport à celui des fissures longues. Cependant très peu de travaux ont été consacrés à l'étude de l'influence de l'environnement sur la propagation des fissures courtes. Lankford [24] a en premier lieu signalé une très faible influence de l'environnement en comparant la croissance de fissures courtes superficielles se propageant à l'air ou sous azote purifié dans un alliage $7075 \mathrm{~T} 651$. Par contre des essais effectués sur un alliage similaire $[25,26]$ à l'air 


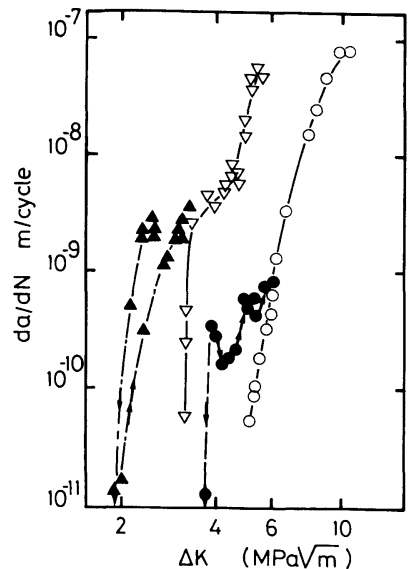

Fig. 1. - Propagation dans l'alliage 2075 T7351 des fissures courtes traversantes $2 \mathrm{D}$ à l'air $(\Delta)$ et sous vide $(\bullet)$ comparativement aux fissures longues air $((\nabla)$ et vide $(0)$ ) dans des éprouvettes CT testées à $R=0,1$ et $35 \mathrm{~Hz}(25)$.

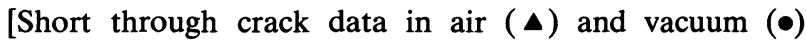
compared to long crack data in air $(\nabla)$ and vacuum $(O)$ for 7075 T651 CT specimens tested at $R=0.1$ and $35 \mathrm{~Hz}$ (25).]

ambiant et sous vide secondaire ont montré une grande influence de l'environnement sur la propagation des fissures 2D (Fig. 1). Cette influence s'avère comparable à celle observée près du seuil pour les fissures longues, et il a été vérifié que le comportement des fissures bidimensionnelles courtes et longues peut être décrit de façon similaire en éliminant les effets de fermeture, c'est-à-dire à l'aide du facteur d'intensité de contrainte effectif $\Delta K_{\text {eff }}$, comme cela a été observé sur d'autres alliages [2729]. Il reste que ce concept ne prend pas en compte l'influence de l'environnement (Fig. 2).

L'objet de la présente étude est de contribuer à une meilleure connaissance de l'influence de l'environnement sur la propagation des fissures courtes 3D dans l'alliage 7075. Deux états de revenus, T651 et $\mathrm{T} 7351$, seront considérés pour tenir compte du rôle éventuel de la microstructure.

\section{Conditions expérimentales.}

La composition de l'alliage 7075 étudié, en \% en poids est de $6,0 \mathrm{Zn}, 2,44 \mathrm{Mg}, 1,52 \mathrm{Cu}, 0,20 \mathrm{Cr}$, 0,16 Fe, 0,07 Si, 0,04 Mn, 0,04 Ti, Al (balance). La tôle utilisée a été trempée après laminage à chaud, puis étirée de $2 \%$. L'état T651 correspond à un traitement de $24 \mathrm{~h}$ à $120^{\circ} \mathrm{C}$ et l'état sur-revenu T7351 à un traitement étagé de $6 \mathrm{~h}$ à $107^{\circ} \mathrm{C}+24 \mathrm{~h}$ à $158^{\circ} \mathrm{C}$. La taille des grains, de forme plate et allongée dans le plan de laminage, est de $600 \times$ $150 \times 40 \mu \mathrm{m}$. L'état T651 présente une résistance maximum (voir tableau I) avec une microstructure caractérisée par la présence de zones GP (1 nm de

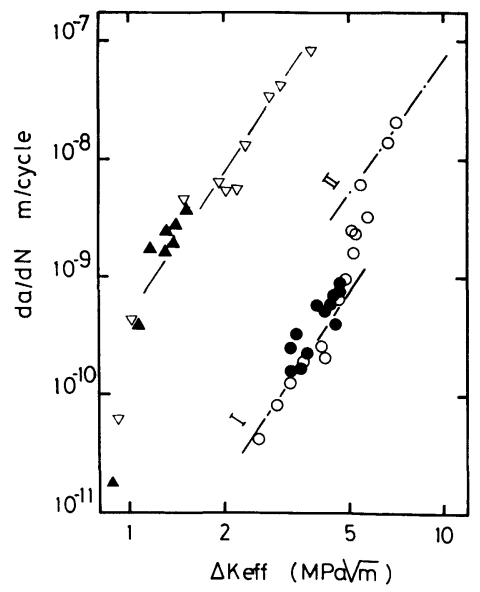

Fig. 2. - Relations $\mathrm{d} a / \mathrm{d} N-\Delta K_{\text {eff }}$ pour les fissures courtes 2D à l'air $(\boldsymbol{\Delta})$ et sous vide $(\bullet)$ comparées à celles obtenues pour les fissures longues (air $(\nabla)$ et vide $(O)$ ) dans le cas des essais de la figure 1 [25].

$\left[\mathrm{d} a / \mathrm{d} N\right.$ vs. $\Delta K_{\text {eff }}$ relationships for short through crack in air $(\Delta)$ and vacuum $(\bullet)$ compared to the effective data for long cracks in air $(\nabla)$ and vacuum $(O)$ corresponding to propagation data presented in figure 1 [25].]

diamètre) et de précipités $\mathrm{M}^{\prime}\left(\mathrm{Mg}_{4} \mathrm{Zn}_{11} \mathrm{Al}\right)$ sous forme de plaquettes $(1,5 \times 5 \mathrm{~nm})$ tous cohérents et cisaillables. La microstructure de l'état sur-revenu se caractérise par la présence de précipités $\mathrm{M}(\mathrm{MgZn} 2)$ et $\mathrm{T}^{\prime}\left(\mathrm{Mg}_{32}(\mathrm{AlZn})_{49}\right)$ semi-cohérents de taille nettement plus grande ( $20 \mathrm{~nm}$ de diamètre) avec de larges zones libres de précipités près des joints de grains.

Tableau I. - Propriétés mécaniques de l'alliage dans les deux états de revenu étudiés.

\begin{tabular}{lccc}
\hline & $\mathrm{R}_{\mathrm{E}}$ & $\mathrm{R}$ & $\mathrm{A} \%$ \\
& $(\mathrm{MPa})$ & $(\mathrm{MPa})$ & \\
\hline $7075 \mathrm{~T} 651$ & 527 & 590 & 11,0 \\
$7075 \mathrm{T7371}$ & 470 & 539 & 11,7 \\
\hline
\end{tabular}

Les essais ont été effectués sur des éprouvettes usinées dans le sens long et dont les dimensions sont indiquées dans la figure 3 . Ils ont été conduits sur une machine hydraulique asservie, à l'air ambiant ou sous vide dans un caisson prévu à cet effet $\left(<5 \times 10^{-4} \mathrm{~Pa}\right)$.

Le calcul du facteur d'intensité de contrainte pour une fissure semi-circulaire de longueur $2 a$, a été effectué à l'aide de la relation suivante :

$$
\Delta K=1,32 \Delta \sigma \sqrt{a} .
$$

Un changement en traction ondulée a été appliqué à une fréquence de $35 \mathrm{~Hz}$ et avec un rapport de charge $R=0,1$. 

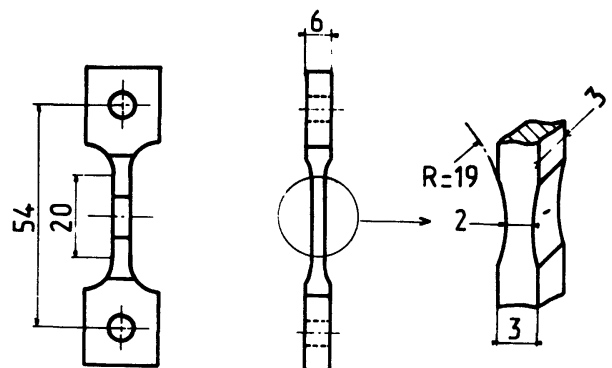

Fig. 3. - Géométrie des éprouvettes pour les essais de propagation de fissures $3 \mathrm{D}$ (en $\mathrm{mm})$.

[Geometry of specimens used for small surface crack tests (all dimensions in $\mathrm{mm}$ ).]

Les fissures courtes naturelles de surface ont été amorcées au niveau de gros précipités intermétalliques (quelques microns de diamètre) en augmentant progressivement la charge. La croissance de la fissure a ensuite été détectée à l'aide de répliques effectuées périodiquement (généralement tous les $10^{4}$ cycles), puis observées au microscope afin de déterminer rétrospectivement l'histoire de la propagation.

L'hypothèse d'une fissure semi-circulaire plane peut se justifier sur la base d'observations faites sur, des éprouvettes rompues en cours de fissuration sur un alliage 7075 T651 [24] et un acier AISI 4140 [30].

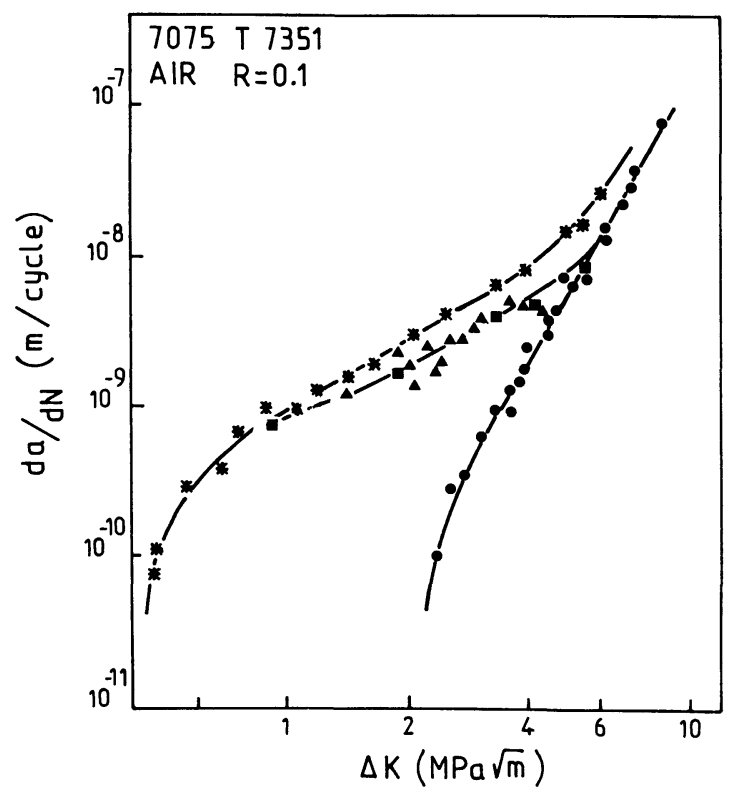

Fig. 4. - $7075 \mathrm{~T} 735$ testé à l'air ambiant à $R=0,1$ et $35 \mathrm{~Hz}$. Propagation d'une fissure courte 3D ( $\square$ ) comparée à celle d'une fissure longue $(\bullet)$ et d'une fissure courte $2 \mathrm{D}$ ( $\Delta$ ) [25]. Relation $\mathrm{d} a / \mathrm{d} N-\Delta K_{\text {eff }}$. pour la fissure longue $(\star)$.

[7075 T7351 tested in ambient air at $R=0.1$ and $35 \mathrm{~Hz}$. Small surface crack data ( $\square$ ) compared to long crack data $(\bullet)$ and $\mathrm{d} a / \mathrm{d} N$ vs. $\Delta K_{\text {eff }}$ data $(\star)$ for long crack. Short through crack data $(\Delta)$ are taken from [26].]
Toutefois, tenant compte des observations faites sur des fissures amorcées au niveau de grosses inclusions (25 à $50 \mu \mathrm{m}$ de diamètre) [27], on considèrera que l'équilibre de la forme semi-circulaire n'est atteint que lorsque la taille de la fissure en surface $(2 a)$ devient au moins trois fois plus grande que celle de l'inclusion ou du précipité du site d'amorçage.

\section{Résultats expérimentaux.}

Alliage SUR-REVENU (T7351). - La figure 4 présente dans un diagramme bilogarithmique exprimant la vitesse $\mathrm{d} a / \mathrm{d} N$ en $\mathrm{m} /$ cycle en fonction du facteur d'intensité de contrainte $\Delta K$ en MPa $\sqrt{\mathrm{m}}$, les mesures de vitesses effectuées pour une fissure 3D se propageant à l'air ambiant dans l'alliage 7075 T7351. Ces résultats sont comparés à ceux obtenus précédemment pour des fissures $2 \mathrm{D}$ courtes [26] et longues [14], et à la relation $\mathrm{d} a / \mathrm{d} N-\Delta K_{\text {eff }}$ obtenue pour une fissure longue à l'aide de mesures de fermeture [14].

Les propagations des fissures courtes $3 \mathrm{D}$ et $2 \mathrm{D}$ paraissent suivre une même loi malgré la grande différence des longueurs initiales (respectivement $12 \mu \mathrm{m}$ et $140 \mu \mathrm{m})$. Il peut en outre être déduit de la courbe $\mathrm{d} a / \mathrm{d} N-\Delta K_{\text {eff }}$ que la propagation de la fissure $3 \mathrm{D}$, tout comme celle d'une fissure courte $2 \mathrm{D}$, commence sans fermeture.

La figure 5 compare les résultats obtenus pour une fissure 3D sous vide à ceux obtenus précédemment

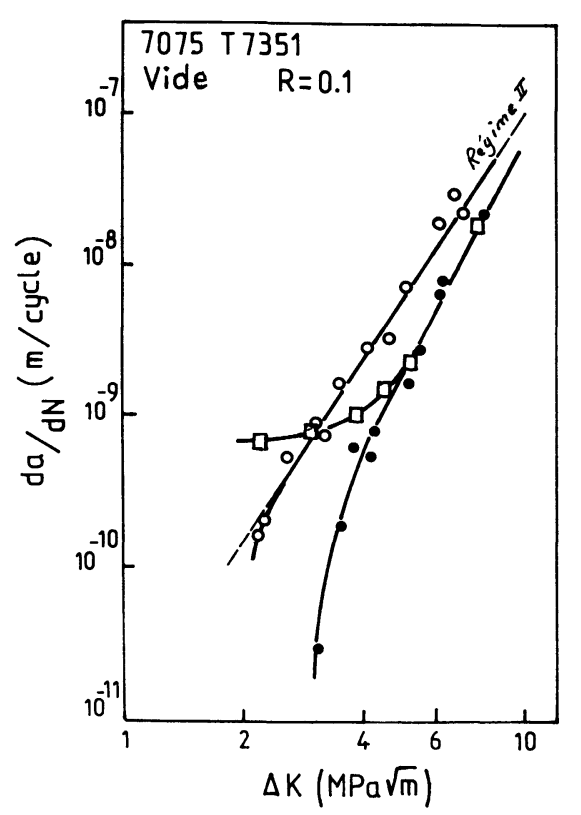

Fig. 5. -7075 T351 sous vide, $R=0,1,35 \mathrm{~Hz}$. Propagation d'une fissure courte 3D ( $\square$ ) comparée à celle d'une fissure longue (•) et à la relation $\mathrm{d} a / \mathrm{d} N-\Delta K_{\text {eff }}$ pour une fissure longue (O).

[7075 T351 tested in vacuum at $R=0.1$ and $35 \mathrm{~Hz}$ small

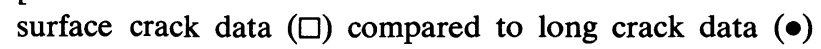
and $\mathrm{d} a / \mathrm{d} N$ vs. $\Delta K_{\text {eff }}$ data for long crack (O).] 
pour une fissure longue. Il en ressort clairement que la propagation sous vide est qualitativement comparable à celle observée à l'air. Mais, quantitativement, la propagation sous vide s'avère bien plus lente qu'à l'air pour un même $\Delta K$ et l'amorçage est obtenu pour une contrainte maximum nettement plus élevée (423 MPa au lieu de $273 \mathrm{MPa}$ à l'air). L'observation d'un début de propagation à un niveau de $\Delta K$ apparemment inférieur à celui que l'on pourrait attendre à partir de la relation $\mathrm{d} a / \mathrm{d} N-\Delta K_{\text {eff }}$, suggère un effet de plasticité localisée [31].

Les microfractographies des figures 6 et 7 montrent que dans les deux environnements, l'amorçage s'effectue au niveau de précipités intermétalliques, et que la propagation en stade II est peu affectée par les joints de grains.

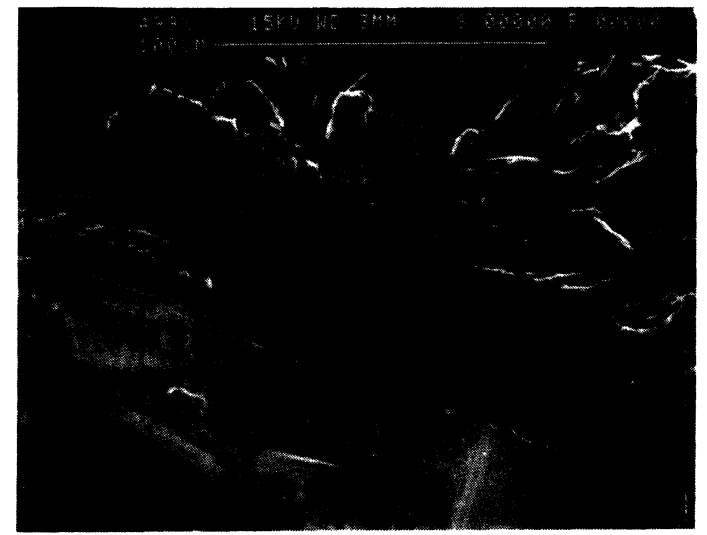

a)

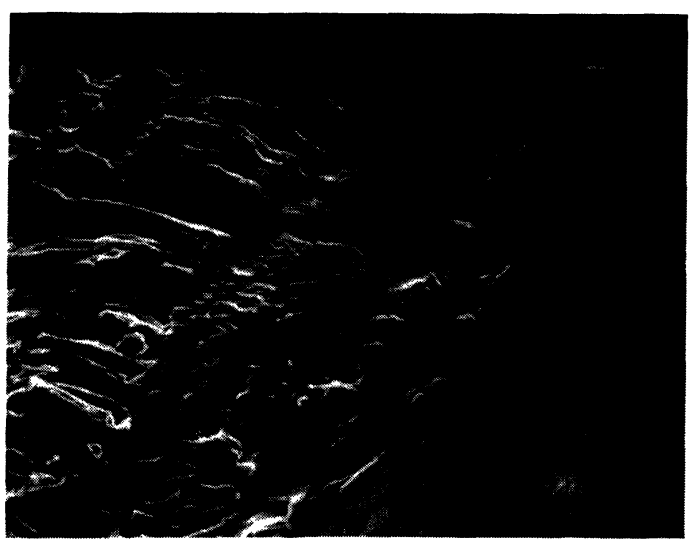

b)

Fig. 6. - Faciès microfractographiques du site d'amorçage des fissures 3D dans les états sur-revenu (a) et T651 (b) testés à l'air ambiant.

[Fracture surface of a small surface crack nearby the nucleation site on the 7075 T7351 alloy (a) and on the 7075 T651 alloy (b) tested in air.]

Alliage 7075 T651 À L'AIR AMBIANT. - Les résultats obtenus à l'air pour une fissure 3D (longueur initiale $=12 \mu \mathrm{m}$, contrainte de $294 \mathrm{MPa}$ ) sont

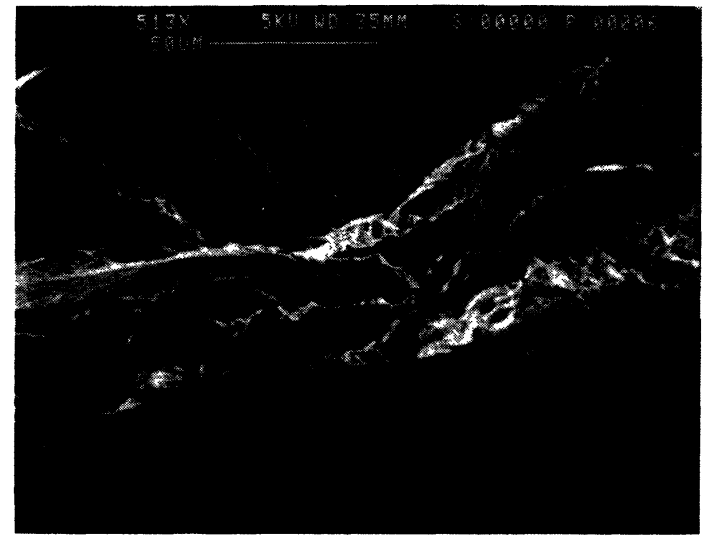

Fig. 7. - Faciès microfractographique de la surface d'une fissure 3D dans l'alliage 7075 T7351 testé sous vide.

[Fracture surface of a small surface crack nearby the nucleation site on the $7075 \mathrm{~T} 7351$ alloy tested in vacuum.]

comparés à ceux obtenus par ailleurs dans trois autres cas (Fig. 8) :

- fissure 3D (Lankford [24]) amorcées sous une contrainte nettement plus élevée (420 MPa) ;

- fissure courte 2D, avec essai de seuil (longueur initiale $=0,2 \mathrm{~mm}$ et longueur au seuil $=0,8 \mathrm{~mm}$ [26]) ;

- fissure longue avec relation $\mathrm{d} a / \mathrm{d} N-\Delta K_{\text {eff }}$ issue des mesures de fermeture [26].

Qualitativement le comportement de la fissure 3D est comparable à celui observé dans l'état T7351 avec un amorçage dans des conditions proches de celles correspondant à la relation $\mathrm{d} a / \mathrm{d} N-\Delta K_{\text {eff }}$, et une loi $\mathrm{d} a / \mathrm{d} N-\Delta K$ recoupant celles obtenues pour les fissures longues. La propagation initiale semble un peu plus lente dans l'état T651, mais reste très comparable pour les valeurs de $\Delta K$ de l'ordre de 2 à $4 \mathrm{MPa} \sqrt{\mathrm{m}}$. L'influence de la microstructure semble donc assez faible. L'aspect microfractographique de la surface de rupture, présenté en figure 6, confirme la similitude observée entre les deux états lorsqu'ils sont testés à l'air ambiant, avec une propagation typiquement en stade II.

Les fissures observées par Lankford ont été amorcées dans des conditions proches de celles correspondant à la relation $\mathrm{d} a / \mathrm{d} N-\Delta K_{\text {eff }}$, les vitesses initiales plus élevées que celles observées ici étant en accord avec un niveau de contrainte également plus élevé. L'existence d'un ralentissement de la propagation immédiatement après l'amorçage pourrait être liée à un effet de plasticité localisée qui n'interviendrait pas dans le cas des essais présentés ici, compte tenu du faible niveau de contrainte initial.

La concordance des courbes de fissuration obtenues pour les fissures 3D, qui, à l'amorçage, sont localisées dans un ou deux grains, et des fissures 2D traversantes, qui «voient " environ une cinquantaine de grains sur leurs fronts, confirme, dans le cas de 


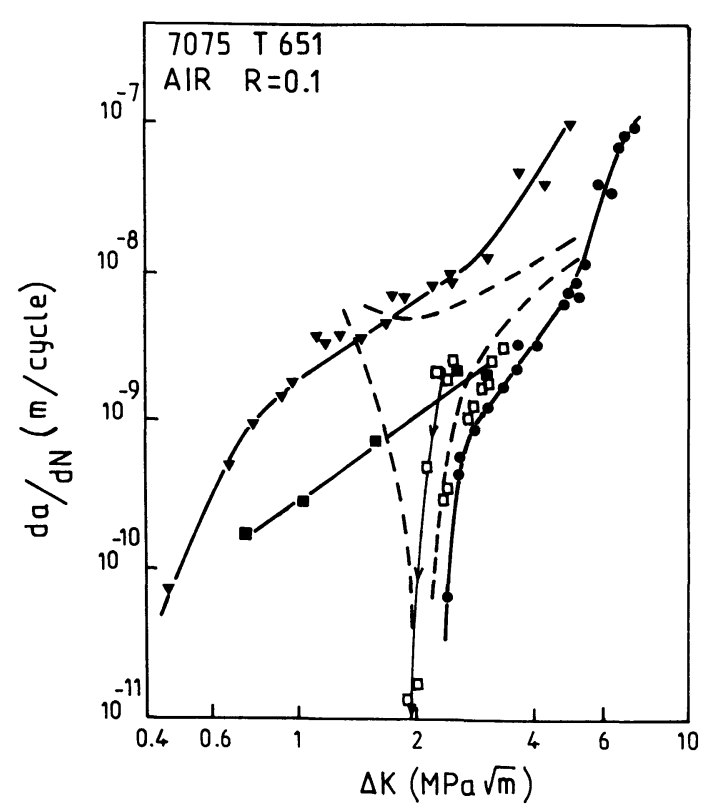

Fig. 8. - 7075 T651 testé à l'air ambiant à $R=0,1$ et $35 \mathrm{~Hz}$. Propagation d'une fissure courte $3 \mathrm{D}(\square)$ comparée à celle d'une fissure longue $(\bullet)$, d'une fissure courte $2 \mathrm{D}$ () [25] et à la courbe $\mathrm{d} a / \mathrm{d} N-\Delta K_{\text {eff }}$ pour les fissures $2 \mathrm{D}$ courtes et longues $(\nabla)$. L'enveloppe des résultats obtenus par Lankford [24] sur ce même alliage est également indiquée (------).

[7075 T651 tested in ambient air at $R=0.1$ and $35 \mathrm{~Hz}$. Small surface crack data ( $\square$ ) compared to long data $(\bullet)$ and $\mathrm{d} a / \mathrm{d} N$ vs. $\Delta K_{\text {eff }}$ relation for the long crack $(\nabla)$. Short through crack data $(\square)$ are taken from figure 1 and Lankford results enveloppe (-----) from [24].]

l'alliage 7075 T651 testé à l'air, l'absence d'influence de la microstructure, comme cela a été observé dans l'alliage sur-revenu à l'air et sous vide.

Alliage 7075 T651 sous VIDE. - La figure 9 présente les résultats obtenus pour les fissures 3D dans l'alliage 7075 T651 testé sous vide. Comme précédemment les résultats sont comparés à ceux obtenus auparavant pour les fissures $2 \mathrm{D}$ courtes et longues [26]. Ces résultats sont qualitativement très différents de ceux obtenus dans les trois autres cas. La propagation initiale, observée à $360 \mathrm{MPa}$ s'avère d'une part, totalement incompatible avec la relation $\mathrm{d} a / \mathrm{d} N-\Delta K_{\text {eff }}$ et d'autre part, beaucoup plus rapide que celle des fissures courtes $2 \mathrm{D}$. Ces résultats montrent que l'effet fissure courte observé ici ne peut pas être expliqué par le seul effet de non fermeture, même en tenant compte de l'éventuel effet de plasticité localisée qui doit être faible compte tenu de la valeur limitée de la contrainte initiale par rapport à la limite élastique $\left(0.68 \sigma_{\mathrm{y}}\right)$.

La figure 10 montre que la morphologie des surfaces rompues est très différente de celles présentées plus haut. On observe des faciès cristallographiques correspondant à une propagation en stade I

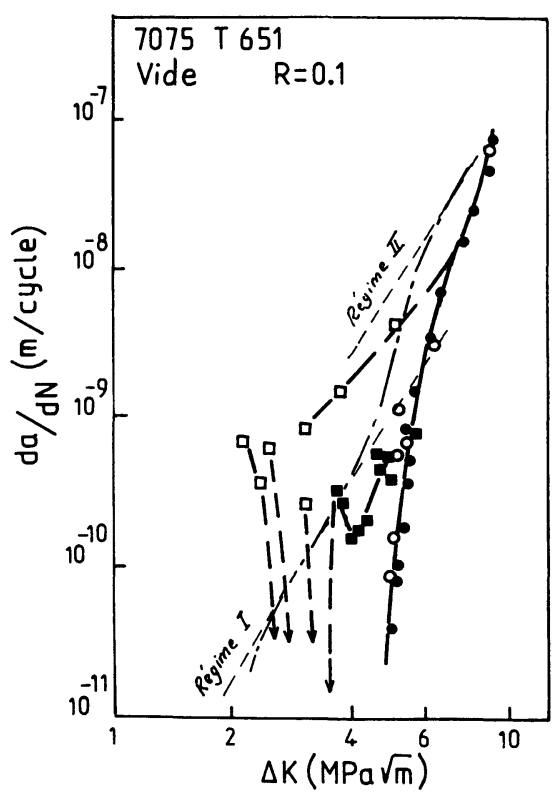

Fig. 9. -7075 T651 testé sous vide à $R=0,1$ et $35 \mathrm{~Hz}$. Propagation d'une fissure courte $3 \mathrm{D}(\square)$ comparée à celle d'une fissure courte 2D (ם) [25] et d'une fissure longue (éprouvettes CT (•) et SEN (O)). Relation $\mathrm{d} a / \mathrm{d} N-\Delta K_{\text {eff }}(-$ -- ---) pour les fissures $2 \mathrm{D}$ courtes et longues.

[7075 T651 tested in vacuum at $R=0.1$ and $35 \mathrm{~Hz}$. Small surface crack data $(\square)$ compared to long crack data on SEN specimen (O) and CT specimen ( $\bullet$ ), and $\mathrm{d} a / \mathrm{d} N$ vs. $\Delta K_{\text {cff }}$ mean curve (-) for long and short through cracks. Short through crack data ( $\square$ ) are taken from figure 1.]

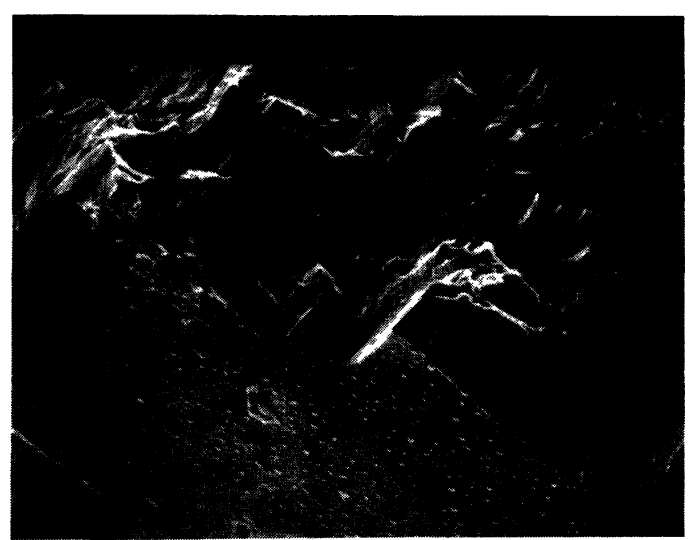

Fig. 10. - Aspect microfractographique de la surface d'une fissure $3 \mathrm{D}$ se propageant en stade $\mathrm{I}$ au voisinage du site d'amorçage dans l'alliage 7075 T651 testé sous vide.

[Stage I propagation of a small surface crack nearby the nucleation site on the 7075 T651 alloy tested in vacuum.]

selon des plans (111). On peut en conclure que dans ces conditions il existe une importante influence de la microstructure sur la propagation des fissures courtes 3D. 


\section{Discussion.}

INFLUENCE DE L'ENVIRONNEMENT. - La moindre résistance à la fissuration en fatigue des alliages légers testés à l'air ambiant comparativement au vide, a été attribuée par différents auteurs à un effet fragilisant de la vapeur d'eau contenue dans l'atmosphère [11-14, 32-35]. L'adsorption de la vapeur d'eau sur les surfaces fraichement rompues en pointe de fissure peut réduire la résistance de l'alliage en diminuant l'énergie de création de surface (effet Rhebinder [34]) comme cela a été proposé [14] pour la propagation à vitesse moyenne $\left(>10^{-8} \mathrm{~m} /\right.$ cycle $)$. Dans de telles conditions, la propagation a été décrite à l'air, comme sous vide, à l'aide d'une relation dérivée du modèle de Weertman $[14,36]$ :

$$
\mathrm{d} a / \mathrm{d} N=A\left(\Delta K_{\text {eff }}\right)^{4} / \mu \sigma^{2} U
$$

où $A$ est un coefficient sans dimension, $\mu$ le module élastique, $\sigma$ la contrainte d'écoulement et $U$ l'énergie nécessaire pour la création d'une unité de surface.

Pour des vitesses suffisamment lentes $\left(>10^{-8} \mathrm{~m} /\right.$ cycle $)$ et inférieures à la vitesse critique définie par Achter [35], un effet de fragilisation du matériau dans la zone plastifiée a été proposé pour expliquer la large influence de l'environnement sur le seuil [14]. Cet effet a été attribué à l'hydrogène produit par dissociation des molécules d'eau adsorbées, puis drainé dans la zone plastifiée par les dislocations se déplaçant sous l'effet du chargement cyclique $[14,25,37,39-41]$. Cette propagation assistée par l'environnement peut être décrite à l'aide d'un modèle du type $\triangle C T O D$ [14] de la forme

$$
\mathrm{d} a / \mathrm{d} N=B\left(\Delta K_{\mathrm{eff}}\right)^{2} / \mu \sigma
$$

où $B$ est un coefficient sans dimension.

On peut voir que la relation (1) est en bon accord avec les résultats sous vide pour l'alliage 7075 T7351 dans une large gamme de vitesses (Fig. 5); par contre deux régimes différents doivent être envisagés pour l'alliage 7075 T651 (Figs. 2 et 10) ; le régime I correspond à une propagation cristallographique en stade I et le régime II correspond au stade II de propagation [14] défini pour l'état T7351.

La propagation cristallographique en stade I dans le 7075 T651 a été reliée à une localisation de la déformation plastique en pointe de fissure selon un seul système de glissements dans chaque grain [14]. Cette localisation est associée à la formation de lignes persistantes de glissement [42] dans la matrice contenant des zones GP et des précipités $\mathbf{M}^{\prime}$ tous cohérents et cisaillables [43]. Dans l'alliage surrevenu, la présence de gros précipités moins cohérents favorise le glissement dévié et l'activation de différents systèmes de glissement [43], ce qui correspond à une propagation en stade II [14].

Les arrêts de propagation observés sous vide dans l'alliage 7075 T651 (Fig. 10) peuvent être attribués à un effet de barrière dû aux joints de grain [24, 44], lorsque qu'un mécanisme de glissement monoplanaire intervient.

Les observations faites à l'air sur ce même alliage suggérent que l'action de l'environnement favorise le stade II de propagation (Fig. 6).

Sur la base des résultats expérimentaux obtenus dans cette étude et de l'analyse faite antérieurement pour les fissures traversantes longues [14] et courtes [25], il peut être admis que la propagation dans l'alliage 7075 des fissures courtes 3D à l'air, observée à des vitesses allant de $2 \times 10^{-10}$ à $10^{-8} \mathrm{~m} /$ cycle, est assistée par l'environnement.

INFLUENCE DE LA PLASTICITÉ LOCALISÉE. - Les fissures $3 \mathrm{D}$ ont été détectées à partir d'une taille $2 a$ de $12 \mu \mathrm{m}$. Dans ce cas la fissure peut être d'une taille inférieure ou égale à celle de la zone plastifiée. Sur la base de mesures expérimentales du $\triangle \mathrm{CTOD}$, Chan [31] suggère que le rapport $p$ du $\triangle \mathrm{CTOD}, \delta$, pour une fissure immergée dans une zone plastifiée, au $\triangle$ CTOD pour une fissure longue, soit égal au rapport du $\triangle \mathrm{CTOD}$ sous chargement monotone $\delta_{\mathrm{m}}$ au $\triangle$ CTOD $\delta_{\text {ssy }}$ dans l'hypothèse d'élasticité linéaire (syy = small scale yielding). En considérant des fissures types Barenblatt et Dugdale, cet auteur propose l'expression suivante de ce rapport:

$p=\frac{\delta_{\mathrm{m}}}{\delta_{\mathrm{ssy}}}=\frac{8}{\pi^{2}}\left(\frac{\sigma_{\mathrm{y}}}{\sigma_{\max }}\right)^{2} \log \left[\sec \left(\frac{\pi}{2} \frac{\sigma_{\max }}{\sigma_{\mathrm{y}}}\right)\right]$

En conséquence, la valeur corrigée de $\Delta K$, $\Delta K^{*}$, tenant compte de l'effet de la plasticité localisée sera :

$$
\Delta K^{*}=p^{1 / 2} \cdot \Delta K
$$

Pour les quatre conditions expérimentales étudiées on obtient ainsi, pour la première mesure de $\Delta K$, les valeurs suivantes de $p^{1 / 2}$ :

\begin{tabular}{ccccc}
\hline Alliage & \multicolumn{2}{c}{7075} & T7351 & \multicolumn{2}{c}{7075 T651 } \\
\hline Environnement & air & vide & air & vide \\
\hline$p^{1 / 2}$ & 1,08 & 1,58 & 1,07 & 1,13 \\
\hline
\end{tabular}

Pour les essais à l'air sur les deux nuances, cet effet reste relativement faible $(<8 \%)$ et donc difficile à détecter expérimentalement. Cette constatation est en accord avec des lois $\mathrm{d} a / \mathrm{d} N-\Delta K$ pratiquement identiques à celles obtenues pour des fissures courtes 2D. L'effet de plasticité localisé étant limité, l'effet fissure courte reste donc essentiellement un effet de fermeture pris en compte à l'aide du concept de $\Delta K_{\text {eff }}$.

Pour l'essai sous vide dans l'alliage sur-revenu, l'amorçage n'a été observé que pour une contrainte 
proche de $95 \%$ de la limite d'élasticité. Dans ce cas la correction proposée par Chan devient très importante. La figure 12 montre que cette correction reporte le point correspondant à la première mesure sur la courbe $\mathrm{d} a / \mathrm{d} N-\Delta K_{\text {eff }}$ Ainsi donc, dans ce cas, le comportement des fissures courtes $3 \mathrm{D}$ et $2 \mathrm{D}$ et des fissures longues peut être décrit comme précédemment à l'aide de la relation $\mathrm{d} a / \mathrm{d} N-\Delta K_{\text {eff }}$, mais après correction [1] de l'effet de plasticité localisée pour les fissures courtes 3D.

Par contre, la figure 12 montre que pour l'alliage 7075 T651 testé sous vide, cette correction [1] ne permet pas à elle seule d'analyser complètement le comportement des fissures 3D.

INFLUENCE DE LA MICROSTRUCTURE. - Très récemment Chan et Lankford ont introduit un concept de «dissimilitude microstructurale » pour les fissures courtes $3 \mathrm{D}$ [45]. Ils considèrent que, lors du premier stade de propagation, la fissure 3D ne voit qu'un seul grain, donc un monocristal. Ils en déduisent une diminution de la valeur de la limite d'élasticité à prendre en considération avec :

$$
\sigma_{\mathrm{ym}}=M \tau_{\mathrm{crss}}
$$

$\sigma_{\mathrm{ym}}=$ limite d'élasticité d'un monocristal

$\tau_{\text {crss }}=$ contrainte critique de cisaillement

$M$ = facteur de Taylor.

Dans le cas d'un polycrystal, on aura :

$$
\sigma_{\mathrm{y}}=\bar{M} \tau_{\mathrm{crss}}
$$

$\sigma_{\mathrm{y}}=$ limite d'élasticité d'un polycristal

$\bar{M} \quad$ = facteur de Taylor pour polycristaux.

Considérant un modèle de superposition dans lequel la fissure $3 \mathrm{D}$ peut être vue comme une fissure respectant les conditions d'application de la Mécanique Linéaire Elastique mais avec un chargement additionnel en tension, ces auteurs proposent une correction [2] de $\Delta K$ pour tenir compte de cet effet lié à la microsctructure telle que :

$$
\Delta K *=q \cdot \Delta K
$$

avec :

$$
\begin{gathered}
q=1+\frac{4 \sqrt{2}}{\pi} \frac{\sigma_{\mathrm{y}}}{\sigma_{\max }} \sqrt{\frac{b}{a}}(1-\Lambda) \\
\Lambda=\frac{M}{\bar{M}}=\frac{\sigma_{\mathrm{ym}}}{\sigma_{\mathrm{y}}} .
\end{gathered}
$$

Les résultats obtenus montrent que la correction [2] n'a pas lieu d'être appliquée dans le cas d'une fissure se propageant en stade II. Dans ce cas, en effet, plusieurs systèmes de glissement (au moins 2) sont activés simultanément et $\Lambda$ est proche de l'unité.

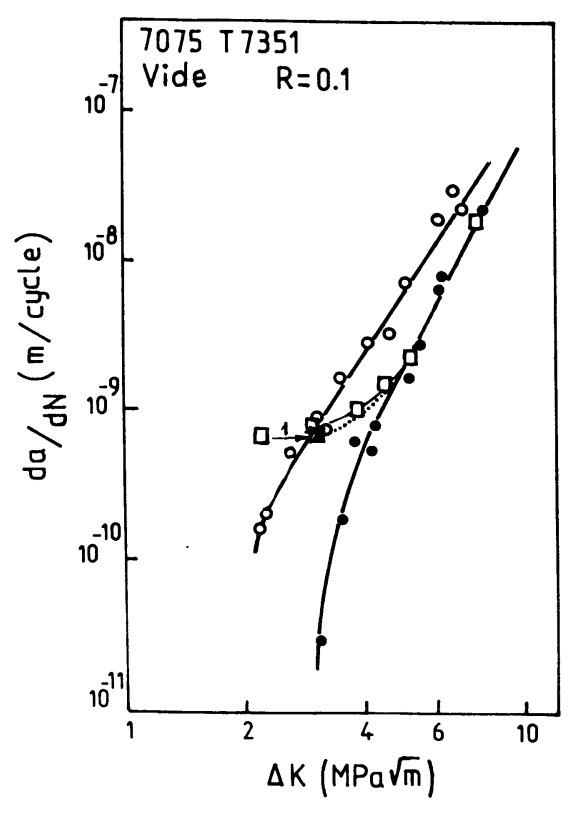

Fig. 11. - Influence de la correction [1] de l'effet de plasticité localisée dans le cas de la figure 5.

[Influence of correction for local plasticity (1) on small surface crack data presented in figure 5.]

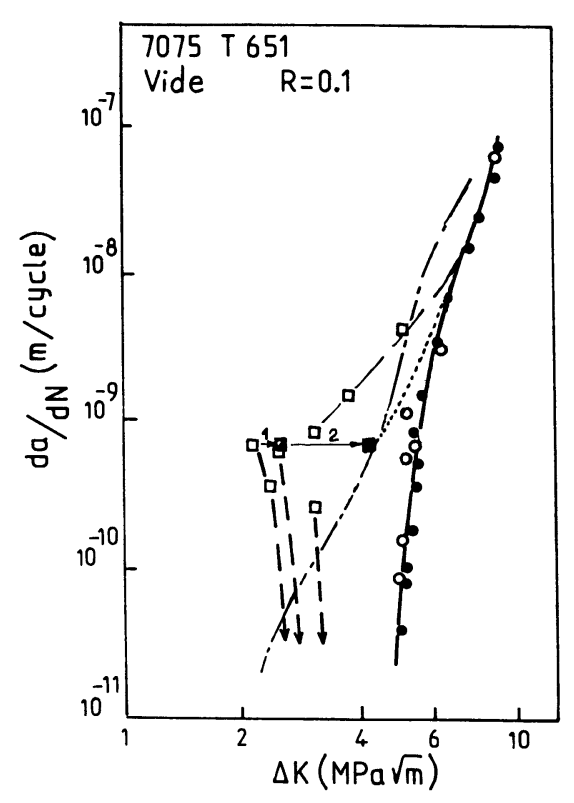

Fig. 12. - Influence des corrections [1] et [2] respectivement des effets de plasticité localisée et de la microstructure dans le cas de la figure 9.

[Influence of correction for local plasticity (1) and microstructural dissimilitude (2) on small surface crack data presented in figure 9.]

Par contre, lorsque la fissure se propage en stade I selon le plan (111) le mieux orienté, donc le plus proche de $45^{\circ}$ par rapport à l'axe de chargement, la correction proposée est nécessaire. La figure 12 montre que les premières mesures se trouvent ainsi 
transférées au voisinage de la courbe $\mathrm{d} a / \mathrm{d} N-\Delta K_{\text {eff }}$ (y compris la correction de plasticité localisée).

CORRECTION DES DIFFÉRENTS EFFETS. - Les travaux antérieurs avaient montré que l'on pouvait décrire le comportement des fissures $2 \mathrm{D}$ courtes et longues à l'aide du facteur d'intensité de contrainte effectif $\Delta K_{\text {eff }}$, donc après correction des effets de fermeture :

$$
\Delta K_{\mathrm{eff}}=U \cdot \Delta K .
$$

Pour les fissures courtes 3D, deux autres facteurs doivent être introduits conduisant à une expression de la valeur de $\Delta K$ réellement appliquée, appelé $\Delta K^{*}$, de la forme :

$$
\Delta K^{*}=p^{1 / 2} \cdot U \cdot \Delta K .
$$

Pour des chargements tels que $\sigma_{\max } / \sigma_{\mathrm{y}}$ soit $<0,6$, $p$ peut être négligé, et pour des fissures se propageant en stade II, $q$ peut également être négligé.

Toutefois aucune de ces corrections ne prend en compte l'influence de l'environnement qui provoque des changements de mécanismes de fissuration qui ne sont pas pris en compte par une telle approche.

\section{Conclusions.}

1. Une forte influence de l'environnement a été mise en évidence sur la propagation des fissures courtes 3D dans un alliage 7075 testé dans les conditions de revenu T651 et T7351. Des vitesses de propagation plus élevées et une résistance à l'amorçage plus faible ont été observées à l'air ambiant comparativement au vide. La plus faible résistance à l'air peut être attribuée à un effet fragilisant de la vapeur d'eau selon un mécanisme décrit précédemment pour les fissures 2D longues et courtes [14, 25].

2. L'augmentation du facteur d'intensité de contrainte pour une fissure immergée dans une zone plastifiée peut être prise en compte à l'aide du modèle de Chan [31].

3. Un important effet de la microstructure a été mise en évidence dans le cas de l'alliage 7075 T651 fissuré sous vide où l'on observe une propagation cristallographique en stade I. Cet effet peut être pris en compte par un modèle proposé par Chan et Lankford [45]. Dans les autres cas, la propagation d'une fissure courte $3 \mathrm{D}$ en stade II apparaît peu sensible à la microstructure.

\section{Bibliographie}

[1] Hudson C. M., Problems of fatigue of metal in vacuum environment, NASA TN D-2563 (1965).

[2] Hudson C. M. and Seward S. K., A Litterature Review and Inventory of the Effects of Environment on the Fatigue Behavior of Metals, Engng Fract. Mech. 8 (1976) 315-322.

[3] KiRby R. B. and BeEVERS C. J., Slow fatigue crack growth and threshold behaviour in air and vacuum of commercial Aluminium alloys, Fat. Engng Structure 1 (1979) 208-215.

[4] Petit J. and Zeghloul A., Gaseous Environmental Effect on Threshold Level in High Strength Aluminium Alloys, Proc. ISFT, Fatigue Threshold, Stockholm, Eds., J. Bäcklund, A. Blom and C. J. Beevers EMAS, Warley (U.K.) A (1981) pp. 563-579.

[5] Petit J., Ranganathan N. and De Fouquet J., Vacuum effect on fatigue crack propagation at low rate, Proc. E.C.F. 3, Fracture and Fatigue, Ed. J. C. Radon (Pergamon Press, London) 1980, pp. 329-337.

[6] Davidson D. L., Incorporating threshold and environmental effects into the damage accumulation model for fatigue crack growth, Fat. Engng Mat. and Struct. 3 (1980) 229-236.

[7] Vasudevan A. K. and Suresh S., Influence of Corrosion Deposits on Near Threshold Fatigue Crack Growth behavior in 2XXX and 7XXX series Aluminium Alloys, Met. Trans. 13A (1982) 2271-2279.

[8] Carter R. D., Lee E. W., Starke E. A., Jr., BEEVERS, C. J., The effect of microstructure and environment on fatigue crack closure of 7475 Aluminium alloy, Met. Trans. 15A (1984) 555-563.

[9] Lindigkeit J., Gysler A. and Lutjering G., The effect of microstructure on the fatigue crack propagation behavior of an $\mathrm{Al}-\mathrm{Zn}-\mathrm{Mg}-\mathrm{Cu}$ alloy, Met. Trans. 12A (1981) 1613-1619.

[10] LANKFORD J. and DAVIDSON D. L., Fatigue crack micromechanisms in ingot powder metallurgy 7XXX aluminium alloys in air and vacuum, Acta Met. 8 (1983) 1273-1284.

[11] Petit J., Bouchet B., Gasc C. and de Fouquet J., A contribution to the study of the influence of environment on the crack growth rate of high strength aluminium alloys in fatigue, Proc. ICF'4, Fracture 1977 (University of Waterloo Press, Canada) M 201 (1977) pp. 867-871.

[12] Petit J., Renaud P. and Violan P., Effects of microstructure on crack growth in a high strength aluminium alloys, Proc. ECF'4, Fracture and the role of microstructure, Eds. K. L. Maurer, F. E. Matzer (EMAS pub., Warley, U.K.) 2 (1982) 426-433.

[13] Renaud P., Violan P., Petit J. and Ferton D., Microstructural Influence on Fatigue Crack Near Threshold in 7075 Alloy, Sc. Met. 16 (1982) 1311-1316.

[14] Petit J., Some aspects of near-threshold crack growth : microstructural and environmental effects, Fatigue Crack Growth Threshold Concepts, Eds. D. Davidson and S ; Suresh (The Metallurgical Society of AIME pub.) 1984, pp. 3-24. 
[15] Vasudevan A. K., and Bretz P. E., Near threshold fatigue crack growth behaviour in $7 \mathrm{XXX}$ and 2XXX alloys : a brief revew, ibid. pp. 25-42.

[16] Starke E. A., Jr., Lin F. S., Chen R. T. and HEIKKENEN H. C., The use of the cyclic stress strain curve and a damage model for predicting fatigue crack growth thresholds, ibid. pp. 43-61.

[17] McEvily A.J. and MinaKawa K., Crack closure and the conditions for fatigue crack propagation, ibid. pp. 517-530.

[18] JatA K. V. and Starke E. A., Jr., Fatigue crack growth and fracture toughness behavior of AlLi-Cu-alloy, Alluminium-Lithium Alloys III, Eds. C. Baker, P. J. Gregson, S. J. Harris and C. J. Peel (The Institue of Metals pub., London) 1986, pp. 247-256.

[19] Petit J., Suresh S., Vasudevan A. K. and MalCOLM R. C., Constant amplitude and postoverload fatigue crack growth in Al-Li alloys, ibid. pp. 257-262.

[20] Tintillier R., YANG H. S., Ranganathan N. and PETIT J., Near threshold fatigue crack growth in a 8090 Lithium containing $\mathrm{Al}$ alloy, 4th International Aluminium Lithium Conference, Eds. G. Champier, B. Dubost, D. Miannay and L. Sabetay (Editions de Physique pub., Paris, France) 1987, pp. 777-784.

[21] Ritchie R. O. and LANKFord J., Small Fatigue Cracks (The Metallurgical Society of AIME pub., Warendale, PA) 1986.

[22] Miller K. J. and DE Los Rios E. R., The behaviour of short fatigue cracks (E.G.F. Pub., London) $\mathrm{n}^{\circ} 1$ (1986).

[23] Pearson S., Initiation of fatigue cracks in commercial aluminium alloys and the subsequent propagation of very short cracks, Eng. Fract. Mech. 7 (1975) 235-247.

[24] LANKFORD J., The effect of environment on the growth of small fatigue cracks, Fat. Engng Mat. Struct. 6 (1983) 15-31.

[25] Petit J. and Zeghloul A., On the effect of environment on short crack growth behaviour and threshold, The behaviour of Short Fatigue Cracks, Eds. K. J. Miller and E.R de Los Rios (E.G.F. Pub., London) $n^{\circ} 1$ (1986) 163-177.

[26] Zeghloul A. and Petit J., $\Delta K_{\text {eff }}$ rationalization of short crack behavior: environmental and microstructural influence, Small Fatigue Cracks, Eds. O. Ritchie and J. Lankford (The Metallurgical Society of AIME, pub.) 1986, pp. 225-238.

[27] Pineau A., Short fatigue crack behavior in relation to thre-dimensional aspects and crack closure effect, Small Fatigue Cracks, Eds. O. Ritchie and J. Lankford (The Metallurgical Society of AIME pub., Warendale, P.A.) 1986, pp. 191212.

[28] JAMES M. R. and MORRIS W. L., Effect of fracture surface roughness on growth of short fatigue cracks, Met. Trans. 14A (1983) 153-155.
[29] MCCARVER J. F. and RITChIE R. O., Fatigue crack propagation threshold for long and short cracks in 95 nickel-base superalloy, Mat. Sci. Engng 55 (1982) 63-67.

[30] London B., Shyne J. C. and Nelson D. V., Small fatigue crack behaviour monitored using surface acoustic waves in quenched and tempered 4140 steel, The behaviour of short fatigue cracks, Eds. K. J. Miller and E. R. de Los Rios (E.G.F. Pub., London) $n^{\circ} 1$ (1986) 537-552.

[31] ChAN K. S., Local crack-tip field parameters for large and small cracks : theory and experiment, Small Fatigue Cracks, Eds. R. O. Ritchie and J. Lankford (The Metallurgical society of AIME pub.) 1986, pp. 407-425.

[32] Wei R. P., Pao P. S., Hart R. G., Weir T. W. and SimMONS G. W., Facture Mechanics and surface chemistry studies of fatigue crack growth in aluminium alloys, Met. Trans. 11A (1980) 151158.

[33] Bowles C. Q., The role of environment, frequency and wave shape during fatigue crack growth in aluminium alloy, Report L.R. 270 (Delf University of Technology pub., The Netherlands) 1978.

[34] Westwood A. R. C. and Ahearn J. S., Adsorption sensitive flow and fracture of solids, Physical Chemistry of the Solid State : Applications to Metals and Compunds, Ed. J. Lacombe (Elsevier pub., Amsterdam) 32 (1984) 65-85.

[35] ACHTER M. R., The adsorption model for environmental effects in fatigue crack growth, Scr. Met. 2 (1968) 525-528.

[36] WeERTMAN J., Theory of fatigue crack growth based on a BCS theory work hardening, Int. J. Fracture 9 (1973) 125-137.

[37] Chakrapany D. G. and Pugh E. N., Hydrogen embrittlement in a $\mathrm{Mg}$-Al alloy, Met. Trans. 7A (1976) 173-178.

[38] JACKo R. J. and DuQueTte D. J., Hydrogen embrittlement of a cyclically deformed high strenght $\mathrm{Al}$ Alloy, Met. Trans. 8A (1977) 1821-1827.

[39] Albrecht J., Bernstein I. M. and Thompson A. W., Evidence of dislocation transport of hydrogen in aluminium, Met. Trans. 13A (1982) 811820.

[40] Zeghloul A. and Petit J., Environmental sensitivity of small crack growth in 7075 aluminium alloy, Fat. Fract. Engng Mater. Struct. 8 (1985) 341-348.

[41] Wei R. P., Ming Gao and Pao P. S., The role of magnesium in CF and SCC of 7000 series Aluminium alloys, Scr. Met. 18 (1984) 1195-1198.

[42] Gerold V., The fatigue properties of precipitationhardened Aluminium alloys, Mat. Sci. Forum 13/14 (1987) 175-194.

[43] SElines R. J., The fatigue behavior of high strength aluminium alloys, Ph. D. Thesis, M.I.T. (1971).

[44] MilleR K. J., The short crack problem, Fat. Engng Mater. Sruct. 5 (1982) 223-232.

[45] Chan K. S. and LANKFord J., Acta Metall. 36 (1988) 193-206. 